\title{
Assessment of Drug Delivery Kinetics to Epidermal Targets In Vivo
}

\author{
M. Hoppel, ${ }^{1}$ M. A. M. Tabosa, ${ }^{1}$ A. L. Bunge, ${ }^{2}$ M. B. Delgado-Charro, ${ }^{1}$ and R. H. Guy ${ }^{1,3}$
}

Received 28 November 2020; accepted 10 February 2021; published online 29 March 2021

\begin{abstract}
It has proven challenging to quantify 'drug input' from a formulation to the viable skin because the epidermal and dermal targets of topically applied drugs are difficult, if not impossible, to access in vivo. Defining the drug input function to the viable skin with a straightforward and practical experimental approach would enable a key component of dermal pharmacokinetics to be characterised. It has been hypothesised that measuring drug uptake into and clearance from the stratum corneum (SC) by tape-stripping allows estimation of a topical drug's input function into the viable tissue. This study aimed to test this idea by determining the input of nicotine and lidocaine into the viable skin, following the application of commercialised transdermal patches to healthy human volunteers. The known input rates of these delivery systems were used to validate and assess the results from the tape-stripping protocol. The drug input rates from in vivo tape-stripping agreed well with the claimed delivery rates of the patches. The experimental approach was then used to determine the input of lidocaine from a marketed cream, a typical topical product for which the amount of drug absorbed has not been well-characterised. A significantly higher delivery of lidocaine from the cream than from the patch was found. The different input rates between drugs and formulations in vivo were confirmed qualitatively and quantitatively in vitro in conventional diffusion cells using dermatomed abdominal pig skin.
\end{abstract}

KEY WORDS: dermal pharmacokinetics; in vitro skin permeation; stratum corneum sampling; topical drug delivery; transdermal delivery systems.

\section{INTRODUCTION}

Modelling 'drug input' into the skin from topical formulations is challenging because the most common sites of action of these products, the viable epidermal and upper dermal compartments, are experimentally difficult to access in vivo. To predict the local (epi)dermal pharmacokinetics and target tissue concentrations, considerable effort has been made to model mathematically the drug transfer from its vehicle into the stratum corneum (SC), its subsequent diffusion across this barrier and its transfer into the viable epidermal/dermal tissue (1). Validation of such predictions requires experimental determinations of the drug input function to the viable skin from the applied formulation. While local drug concentrations in the skin can be measured using spectroscopic techniques (e.g. Raman/IR) or by microdialysis/microperfusion (25 ), these approaches are limited by sensitivity issues, on the one hand, and by considerable technical demands, on the other.

An alternative method to determine the concentration of an active entity in the viable epidermis is based on the measurement of

\footnotetext{
${ }^{1}$ Department of Pharmacy \& Pharmacology, University of Bath, Claverton Down, Bath, BA2 4LZ, UK.

${ }^{2}$ Department of Chemical and Biological Engineering, Colorado School of Mines, Golden, Colorado 80401, USA.

${ }^{3}$ To whom correspondence should be addressed. (e-mail: r.h.guy@bath.ac.uk)
}

drug uptake from a formulation into and elimination of the drug from the SC by tape-stripping, as described recently for acyclovir (6).

The idea behind this approach was first indicated and justified through mathematical modelling (7), and further illustrated in later publications on diclofenac (8) and metronidazole (9). Specifically, the clearance phase, where the drug diffuses from the SC into the deeper layers of the viable skin below, provides valuable information about a topical drug's input function into the viable tissue (6-9). It follows that the mass of drug in the viable tissue depends on the flux of drug from the SC and the rate of its subsequent transfer to the presystemic blood compartment. Therefore, measurement of drug clearance from the SC provides information about topical drug input kinetics into the epidermis/dermis (7).

Hence, for drugs with their site of action in the viable epidermis, the SC sampling methodology, as recently described (6-9), is targeted at eliciting information directly pertinent to the assessment of topical bioavailability (and by extrapolation, eventually, to bio(in)equivalence between different formulations). As such, the new approach to the interpretation of SC sampling data responds directly to an early and still-voiced criticism of tape-stripping that it cannot provide a useful metric related to the rate and extent of drug delivery to sites of action that are found elsewhere in the skin (such as the viable epidermis). 
Of course, the 'input' function only provides part of the information needed to estimate a drug's concentration in the sub-SC compartment and that requires knowledge of the local clearance too (and may be even more complicated for drugs subject to epidermal metabolism). However, there are models and associated algorithms for estimating 'dermal clearance' by the local microcirculation $(10,11)$ and these can be used, together with the delivery rate of drug from SC obtained from tape-stripping experiments, to calculate a concentration in a viable skin compartment-the $C^{*}$ value as described by Higuchi et al. many years ago (12-16).

The aim of this study is first to validate the in vivo SC sampling method using transdermal drug delivery systems for nicotine and lidocaine. Although drugs administered from conventional patches are not typically designed to target structures within the skin, the active entities must pass through the epidermis/dermis en route to the systemic compartment (17). For all approved transdermal patches, the labelling specifically includes information on the drug input rate at steady-state (in amount per unit time) and permits, therefore, an investigation of whether these values can be duplicated using the SC tape-stripping protocol in healthy human volunteers (7). Having established a suitable protocol, the input kinetics of lidocaine from a marketed cream formulation (for which the clinical indication is different from that of the patch (18)) into the viable skin are then assessed. The cream represents a typical topical product, of course, that undergoes a significant 'metamorphosis' (19) during and immediately after its application to the skin, complicating thereby the drug uptake and clearance kinetics into and out of the SC. Finally, to complement the method validation, the in vivo experiments are replicated, as far as is possible, in more conventional in vitro permeation tests using abdominal pig skin as a recognised model membrane (20) for the human counterpart.

\section{MATERIALS AND METHODS}

\section{Materials}

Disodium hydrogen phosphate was purchased from Acros Organics (Geel, Belgium), potassium dihydrogen phosphate from Fisher Scientific (Loughborough, UK), acetonitrile and methanol from VWR Chemicals (Lutterworth, UK). Other solvents, HPLC reagents, lidocaine and (-)-nicotine were obtained from Sigma-Aldrich (Gillingham, UK). Nicotinell® $7 \mathrm{mg} / 24 \mathrm{~h}$ patches (Novartis Consumer Health, Camberley, UK) were bought from Boots UK Limited (Nottingham, UK). Versatis ${ }^{\circledR} 5 \mathrm{mg}$ medicated plaster was from AAH Pharmaceuticals Ltd (Coventry, UK). LMX4 (Lidocaine $4 \% \mathrm{w} / \mathrm{w}$ cream) was purchased from HI Weldrick Ltd (Doncaster, UK).

\section{Methods}

\section{In Vivo Experiments}

Subjects. Eighteen healthy volunteers with no history of dermatological disease participated in the study. Six volunteers were enrolled for each treatment: three males and three females (age range 25-34 years) for the nicotine patch; two males and four females (age range 25-34 years) for the lidocaine patch; three males and three females (age range 2528 years) for the lidocaine cream. The different protocols were approved by the Research Ethics Approval Committee for Health (REACH) of the University of Bath: REACH 15/ 16112 (nicotine) and REACH 16/17 006 (lidocaine). Informed consent was obtained from each subject.

SC Sampling. This study followed a published SC sampling method [7] with a few modifications. One hour before application, the volunteers' forearms were cleaned with a mild soap solution (Cussons Carex complete antibacterial handwash, PZ Cussons, Manchester, UK), rinsed thoroughly with warm water and dried. The same formulation was then applied to both forearms providing duplicate measurements in each volunteer, as described in further detail below. In the earlier study (7), the number of tapestrips required to collect most of the SC was determined by periodic measurements of transepidermal water loss (TEWL) of each treated site; in contrast, here, the number of tapestrips applied to all treated sites for each subject was the same and was based on the average number of tapes required for TEWL (measured with an AquaFlux AF102, Biox Systems Ltd., London, UK) to surpass $60 \mathrm{~g} \mathrm{~m}^{-2} \mathrm{~h}^{-1}$ (or a maximum of 30 tape-strips) for the two untreated (blank) sites on that subject. This was a practical necessity for the nicotine study because the occlusive nature of these patches interfered with the TEWL readings. The lidocaine study, which was conducted after the nicotine experiments, followed the same procedure, except that the TEWL was determined immediately before and after the tape-stripping of each treated site. TEWL measurements of the untreated site were performed before tape-stripping, then occasionally as tape-stripping proceeded, and after 30 strips if the TEWL did not exceed $60 \mathrm{~g} \mathrm{~m}^{-2} \mathrm{~h}^{-1}$ first. Typically, 20-30 tape-strips were required.

The amount of SC on the tapes removed from treated and untreated sites was determined gravimetrically using a microbalance (SE-2F, precision $0.1 \mu \mathrm{g}$; Sartorius AG, Göttingen, Germany) after being discharged of static electricity (R50 discharging bar with ES50 power supply from Eltex Electrostatic GmbH, Weil am Rhein, Germany). From the area tape-stripped and the known density $\left(\sim 1 \mathrm{~g} \mathrm{~cm}^{-3}\right)$ of the SC (21), the mass on each tape was converted to the corresponding thickness removed.

Nicotine. One Nicotinell ${ }^{\circledR} 7 \mathrm{mg} / 24 \mathrm{~h}$ patch was applied on each forearm of the volunteers $(n=6)$. After $2 \mathrm{~h}$, both patches were removed, leaving no detectable residue on the skin, and the application sites were demarcated into three equal areas of $1.8 \mathrm{~cm}^{2}$ using a template cut from Scotch Book tape 845 (3M, St. Paul, MN, USA) (Fig. 1a). One area was tape-stripped immediately ('uptake'), a second at $1.5 \mathrm{~h}$ after patch removal ('1.5-h clearance') and the third at 3-h postpatch removal ('3-h clearance'). Pieces of the same adhesive tape (that were larger than the stripped area) were used to remove the SC with a standardised procedure (7). The template ensured that the application of sequential tapestrips was made on exactly the same location and was designed so that areas of treated skin, which had not yet been stripped, remained unoccluded (Fig. 1b). Because patch adhesion was less consistent at the very edges of the patch, the template ensured that tape-stripping here was avoided (Fig. 1c). All tape-stripping was performed by a single investigator. 

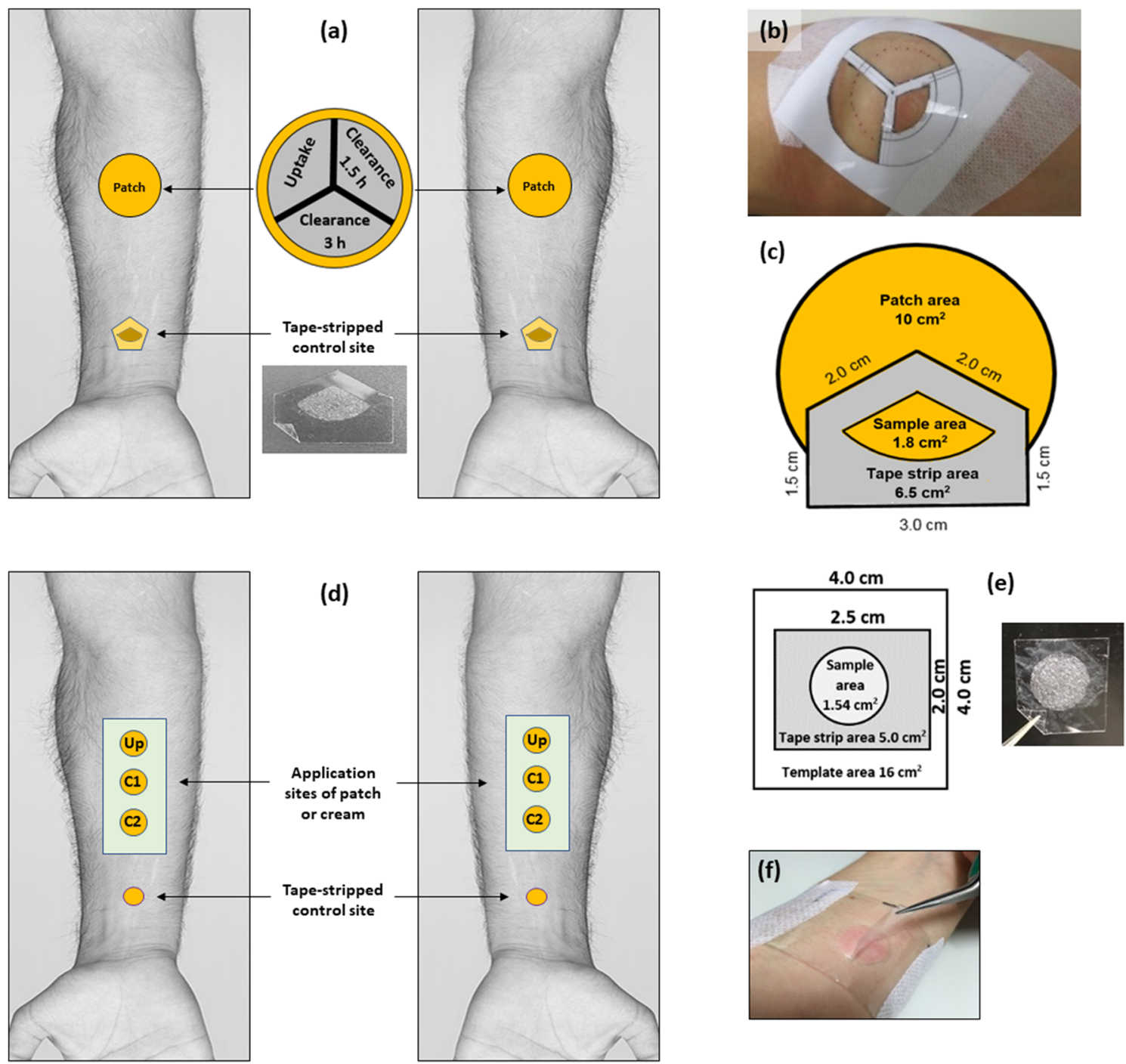

Fig. 1. Pictorial representation of the in vivo SC sampling experiments for nicotine (panels $\mathbf{a}, \mathbf{b}$ and $\mathbf{c}$ ) and lidocaine (panels d, e and $\mathbf{f}$ ). See text for details

Having collected SC at the 'uptake' site immediately after patch removal, the two 'clearance' sites were protected with a rectangular frame $(5.5 \mathrm{~cm} \times 5.5 \mathrm{~cm})$ made of self-stick adhesive pads (Dr. Scholl Pressure Point Foam Padding, Slough, UK) and covered with a non-occlusive mesh (ultra stiff plastic canvas, 7 mesh, Darice ${ }^{\circledR}, \mathrm{OH}$, USA) that was held in place with Mefix (Molnlycke, Oldham, UK). At $1.5 \mathrm{~h}$ after patch removal, the dressing was removed and SC from this first 'clearance' site was then collected in exactly the same manner as that from the 'uptake' area. At $3 \mathrm{~h}$, the procedure was repeated at the second 'clearance' site.

Lidocaine. To a first group of six volunteers, a Versatis ${ }^{\circledR}$ $5 \%$ medicated plaster (or 'patch'), equivalent to $5 \mathrm{mg} \mathrm{cm}^{-2}$ of lidocaine, was applied to three sites on each forearm (Fig. 1d). Patches were worn overnight for $12 \mathrm{~h}$ and were then removed. The treated skin areas were subsequently cleaned quickly with a $70 \%$ isopropyl alcohol wipe (Sterets®, Molnlycke) to completely remove any residual patch material on the skin. In a second cohort of 6 volunteers, both forearms were treated (at a dose consistent with the Summary of
Product Characteristics (22)) with $150 \mathrm{mg} \mathrm{cm}^{-2}$ of LMX4 Lidocaine $4 \% \mathrm{w} / \mathrm{w}$ cream, equal to $6 \mathrm{mg} \mathrm{cm}^{-2}$ of drug, for $1 \mathrm{~h}$. Removal of residual formulation on the skin involved first dry-wiping with absorbent tissue before cleaning with isopropyl alcohol as for the patch. The selected application times of the two formulations reflected the dosing instructions in the corresponding patient information leaflets.

Demarcation of $1.5 \mathrm{~cm}^{2}$ areas of each application site was then made using a template cut from Scotch Book tape 845 (3M) (Fig. 1e). The template ensured that SC collected during the repeated, sequential tape-stripping all originated from exactly the same location. The tape-strips used to remove the SC were much larger than the treated area of SC (Fig. 1e and f). On each arm, for both formulations, one site was tape-stripped immediately ('uptake' (Up)), a second at $4 \mathrm{~h}$ after patch removal ('4-h clearance' (C1)) and the third $8 \mathrm{~h}$ post-patch removal ('8-h clearance' (C2)) (Fig. 1d). Between formulation removal of the two 'clearance' sites (C1 and $\mathrm{C} 2$ ), the skin was protected with a light, nonocclusive gauze (Boots, Nottingham, UK). Again, all tapestripping was performed by a single investigator. 
Drug Extraction from SC Tape-Strips. Nicotine was extracted from the SC removed on each tape-strip by shaking overnight with $1.5 \mathrm{~mL}$ of the mobile phase used for the subsequent HPLC analysis: phosphate buffer 10 mM, pH 7.4/ acetonitrile/methanol, 30:35:35 v/v. The efficiency of nicotine extraction was determined using tape-strips with adhering SC that had been 'spiked' with a known amount of the drug; the mean recovery $( \pm \mathrm{SD})$ was $96.8 \pm 3.5 \%(n=3)$.

Lidocaine was extracted from SC on groups of 2 to 4 tape-strips, which were rolled, placed into small vials with 3 $\mathrm{mL}$ of the same HPLC mobile phase used for nicotine extraction and subjected to 20 min of ultra-sonication (Clifton Ultrasonic Water Bath, Nickel-Electro Ltd., Weston-superMare, UK). The efficiency of lidocaine extraction was assessed in the same way as that used for lidocaine; the mean recovery $( \pm \mathrm{SD})$ was $97.9 \pm 2.0 \%(n=3)$.

\section{In vitro Experiments}

Abdominal pig skin was obtained post-sacrifice without having been exposed to the normal high-temperature cleaning procedure. Skin was dermatomed (Zimmer®, Warsaw, IN, USA) to a nominal thickness of $750 \mu \mathrm{m}$ and stored frozen. Before use, the skin was thawed, and any visible hairs were trimmed with scissors.

Nicotine. The nicotine patch was cut into circular discs (diameter of $1.4 \mathrm{~cm}$, surface area $1.54 \mathrm{~cm}^{2}$ ) and adhered to the external side of the skin (pressure having been applied with a custom-made roller passed 10 times in two directions to ensure complete adhesion). The skin was mounted in a glass Franz diffusion cell having an internal diameter of $1.4 \mathrm{~cm}$ (PermeGear, Inc., Bethlehem, PA, USA). The receptor chamber had been filled with phosphate-buffered saline solution (10 mM, pH 7.4, $7.1 \mathrm{~mL}$ ). The receptor solution was stirred magnetically at a constant speed of $500 \mathrm{rpm}$ and the temperature was maintained at $37 \pm 1{ }^{\circ} \mathrm{C}$ by circulating warmed water through a jacket surrounding the cell.

The cumulative delivery of nicotine into the receptor solution was determined following a single 5-h application of the nicotine patch. Aliquots $(1 \mathrm{~mL})$ were withdrawn at 1,2 , 3.5 and $5 \mathrm{~h}$, then immediately replaced with the same volume of fresh receptor solution. Samples were filtered (Cronus syringe filter, nylon, $4 \mathrm{~mm}, 0.45 \mu \mathrm{m}$, LabHut, Gloucester, UK) and the concentration of nicotine in the samples was quantified by HPLC as described below. Six replicates were performed for each experiment.

The amount of drug permeated in each sampling interval was calculated from its concentration in the receptor solution and the volume of receptor chamber. The cumulative amount of drug permeated as a function of time was calculated.

Lidocaine. The lidocaine patch was cut into circular discs (diameter of $1.4 \mathrm{~cm}$, surface area $1.54 \mathrm{~cm}^{2}$ ) and firmly applied to the external side of the skin (as for nicotine). The cream was applied in the same amount as in vivo $\left(150 \mathrm{mg} \mathrm{cm}^{-2}\right.$ of cream) using a cotton bud. The skin was mounted in Franz diffusion cells operated as for nicotine except that the temperature in these experiments was controlled by placing the cells in an oven at $32^{\circ} \mathrm{C}$. The cumulative amount of lidocaine delivered post-drug application was determined by sampling (with replacement) of the receptor solution at 2, 4, $6,8,10$ and $12 \mathrm{~h}$ for the patch, and at $1,3,5,7$ and $9 \mathrm{~h}$ for the cream; seven replicates were performed for each formulation. All samples were filtered (Cronus syringe filter, nylon, $4 \mathrm{~mm}$, $0.45 \mu \mathrm{m}, \mathrm{LabHut}, \mathrm{UK})$ and the concentration of lidocaine in the samples was quantified by HPLC as described below. The steady-state flux and lag time (intercept of the time axis) were estimated from the linear gradient of the cumulative amount of drug penetrated versus time profile using measurements at times that exceeded $2.4 \times$ the lag time for each cell (23) or from the last sampling interval (for some experiments with the patches) when $2.4 \times$ the lag time exceeded $10 \mathrm{~h}$.

\section{Analysis and Interpretation of In Vivo Skin Uptake and Penetration Data}

The amount of drug in the SC was measured immediately after the applied formulation (patch or cream) is removed, the socalled uptake, and then after two periods of 'clearance'. In a first approach, the SC is considered to be a well-stirred compartment, from which drug is eliminated with 1st-order kinetics described by a rate constant $\beta$. The 'clearance' of drug from the SC can therefore be described as follows:

$\mathrm{dM}_{\mathrm{SC}} / \mathrm{dt}=-\beta \times \mathrm{M}_{\mathrm{SC}}$

where $\mathrm{M}_{\mathrm{SC}}$ is the amount of drug in the SC at ('clearance') time $t$ after formulation removal. The relevant, initial boundary condition is that, at $t=0$ (i.e. when uptake is finished), $\mathrm{M}_{\mathrm{SC}}=\mathrm{M}_{\mathrm{UB}}$ where $\mathrm{M}_{\mathrm{UP}}$ is the drug amount in the $\mathrm{SC}$ at the end of the uptake period. Equation (1) may then be solved:

$\mathrm{M}_{\mathrm{SC}}=\mathrm{M}_{\mathrm{UP}} \times \mathrm{e}^{-\beta \mathrm{t}}$

and

$\ln \mathrm{M}_{\mathrm{SC}}=\ln \mathrm{M}_{\mathrm{UP}}-\beta \times \mathrm{t}$

It follows that the in vivo experiments provide measurements of $\mathrm{M}_{\mathrm{SC}}$ at three 'clearance' times, i.e. $0,1.5$ and $3 \mathrm{~h}$ following application of the nicotine patch, and 0,4 and $8 \mathrm{~h}$ following administration of both the lidocaine patch and cream. Linear regression of the geometric mean values of the duplicate measurements at uptake and two clearance times yields values of $M_{U P}$ and $\beta$ from the intercept and from the intercept and slope; these parameters can then be used to 'estimate' for each subject the input rate $\left(R_{1}\right)$ of drug from the SC into the underlying viable skin compartment at the moment the 'uptake' finished, i.e.

$R_{1}=-\left(\mathrm{dM}_{\mathrm{SC}} / \mathrm{dt}\right)_{\mathrm{t}=0}=\beta \times \mathrm{M}_{\mathrm{UP}}$ 
Assuming $\beta$ is constant over the entire time of clearance. The input rate can also be estimated for just the shorter clearance interval $\left(R_{1, \mathrm{CL} 1}\right)$ as

$R_{1, \mathrm{CL} 1}=\beta_{\mathrm{CL} 1} \times \mathrm{M}_{\mathrm{UP}}=\left[\ln \left(\mathrm{M}_{\mathrm{UP}} / \mathrm{M}_{\mathrm{CL}}\right) / \Delta \mathrm{t}\right] \times \mathrm{M}_{\mathrm{UP}}$

where $\mathrm{M}_{\mathrm{UP}}$ and $\mathrm{M}_{\mathrm{CL}}$ are the geometric mean values of the duplicate measurements determined respectively at uptake and at clearance for $\Delta \mathrm{t}=1.5 \mathrm{~h}$ for nicotine and $4 \mathrm{~h}$ for lidocaine. If $\beta$ is not the same for both the shorter and longer clearance times, then we can expect that $\mathrm{R}_{1, \mathrm{CL} 1}$ will be a better estimate of the input rate to the viable tissue before the drug product is removed from the skin surface.

A second approach that has been reported in the literature $(6,8,9)$ estimates the input rate $\left(R_{2}\right)$ from the difference between the amounts of drug in the SC after 'uptake' $\left(\mathrm{M}_{\mathrm{UP}}\right)$ and after a period of 'clearance' $\left(\mathrm{M}_{\mathrm{CL}}\right)$ divided by the time elapsed between the two measurements $(\Delta \mathrm{t})$ :

$R_{2}=\left(\mathrm{M}_{\mathrm{UP}}-\mathrm{M}_{\mathrm{CL}}\right) / \Delta \mathrm{t}$

Calculation of $R_{2}$ for the shorter clearance time should provide the closest comparison to the input rate before the drug product is removed and to the estimated input rate by the first approach (i.e. $R_{1}$ if $\beta$ is constant, and $R_{1, \mathrm{CL} 1}$ if $\beta$ is not the same constant for the shorter and longer intervals). For the first clearance interval, $R_{2}$ depends on the fraction of mass remaining in the $\mathrm{SC}$ as follows:

$R_{2}=\mathrm{M}_{\mathrm{UP}}\left(1-\mathrm{M}_{\mathrm{CL} 1} / \mathrm{M}_{\mathrm{UP}}\right) / \Delta \mathrm{t}$

It follows that the calculated values of $R_{2}$ and $R_{1, \mathrm{CL} 1}$ (Eq. 5) will be equivalent whenever the fraction that cleared from the $\mathrm{SC}$ (i.e., $1-\mathrm{M}_{\mathrm{CL} 1} / \mathrm{M}_{\mathrm{UP}}$ ) is small. The clearance rate from the SC slows as the drug amount in the SC decreases. As a result, even if $\beta$ does not change, the average flux over the interval (represented by $R_{2}$ ) decreases, whereas the clearance rate represented by $R_{1, \mathrm{CL} 1}$ does not because it is estimated from the drug amount in the SC when clearance begins.

\section{HPLC Analysis}

Nicotine was quantified by HPLC (LC-2010, AHT, Shimadzu, Milton Keynes, UK) with UV detection (206 nm) $(24,25)$ using a mixture of phosphate buffer $10 \mathrm{mM}$, pH 7.4: acetonitrile:methanol $(30: 35: 35 \mathrm{v} / \mathrm{v})$ as the mobile phase. Samples were filtered (Cronus syringe filter, nylon, $4 \mathrm{~mm}$, $0.45 \mu \mathrm{m}$ ) prior to analysis and run on a C18 column (Kya Tech, London, UK) with a precolumn (Phenomenex, Macclesfield, UK). The flow rate was $1 \mathrm{~mL} \mathrm{~min}^{-1}$ and the column oven temperature was $25{ }^{\circ} \mathrm{C}$. Runtime was $4 \mathrm{~min}$ and the nicotine retention time was $\sim 3.1 \mathrm{~min}$. A calibration curve was established between 10 and $0.16 \mu \mathrm{g} \mathrm{mL}^{-1}$ with $R^{2}=$ 0.999. The limits of detection (LOD) and quantification (LOQ) were $0.05 \mu \mathrm{g} \mathrm{mL}^{-1}$ and $0.17 \mu \mathrm{g} \mathrm{mL}^{-1}$, respectively, and correspond to $0.04 \mu \mathrm{g} \mathrm{cm}^{-2}$ and $0.14 \mu \mathrm{g} \mathrm{cm}^{-2}$ of drug per tape-strip.
Lidocaine was also quantified by HPLC (Summit, Dionex, Swindon UK) with UV detection $(240 \mathrm{~nm})$ using a mixture of phosphate buffer $10 \mathrm{mM}, \mathrm{pH}$ 7.4:methanol (30:70 $\mathrm{v} / \mathrm{v}$ ) as the mobile phase. All samples were filtered (Cronus syringe filter, nylon, $4 \mathrm{~mm}, 0.45 \mu \mathrm{m}$ ) prior to analysis. In vitro permeation test samples were run on a C18 $150 \times 4.6 \mathrm{~mm}$ column (Kya Tech) at $1 \mathrm{~mL}$ min $^{-1}$ flow rate, with a $50 \mu \mathrm{L}$ injection volume and an oven temperature of $30^{\circ} \mathrm{C}$. In vivo tape-stripping samples were run on a Kinetex ${ }^{\circledR}$ C18 $250 \times$ $4.6 \mathrm{~mm}$ column (Phenomenex) at $0.9 \mathrm{~mL} \mathrm{~min}^{-1}$ flow rate, with a $10 \mu \mathrm{L}$ injection volume at $40^{\circ} \mathrm{C}$. Runtime was $8.5 \mathrm{~min}$ and the lidocaine retention time was $\sim 6.5 \mathrm{~min}$. For both in vitro and in vivo experiments, calibration curves between 10 and $0.16 \mu \mathrm{g} \mathrm{mL}{ }^{-1}$ with $R^{2}=0.999$ were produced. LOD and LOQ were $0.13 \mu \mathrm{g} \mathrm{mL}^{-1}$ and $0.38 \mu \mathrm{g} \mathrm{mL}^{-1}$, respectively, in vitro, and $0.08 \mu \mathrm{g} \mathrm{mL}^{-1}$ and $0.24 \mu \mathrm{g} \mathrm{mL}^{-1}$, in vivo. The in vivo values correspond to $0.16 \mu \mathrm{g} \mathrm{cm}^{-2}$ and $0.48 \mu \mathrm{g} \mathrm{cm}^{-2}$ of drug per group of tape-strips.

A few detectable observations for nicotine (in vivo) and lidocaine (in vitro) were below the LOQ; these were assigned the value zero. The maximum possible effects of this choice on the in vivo nicotine results (calculated by comparing with results that assigned the $\mathrm{LOQ}$ value to all determinations less than the LOQ) were $2.5 \%$ at most for the drug amount in an individual volunteer and less than $1 \%$ for the mean values of drug mass, $\beta$ and input rates. The same analysis of the in vitro lidocaine results showed no discernible difference in the results reported.

\section{Statistics}

All statistical analyses were performed with GraphPad Prism 5.01 (GraphPad Software, Inc., La Jolla, CA, USA) with $p<0.05$ as level of significance. In the in vivo study, the geometric mean of the duplicate measurements in each subject were calculated, and averages for all subjects are reported as the geometric mean and $90 \%$ confidence intervals. The specific statistical tests for comparison of experimental observations are identified in the respective 'Results' section that follows.

\section{RESULTS AND DISCUSSION}

The duplicated measurements of nicotine and lidocaine levels in the SC of each subject, in uptake and clearance for the three delivery systems studied, are summarised in Fig. 2; the complete dataset comprising the drug profiles as a function of SC depth is in Fig. S1 (Supplementary Information). The reproducibility of the approach was good with only two statistically significant outliers identified (the higher values for nicotine subject 3 in uptake and lidocaine subject 9 in clearance at $8 \mathrm{~h}$; Grubb's test, $p<0.05$ (26)).

Table I summarises the information in Fig. 2 and shows-as expected-that, once the delivery system is removed, the drug is progressively cleared from the skin. The amount of drug in the SC after the first interval of clearance was reduced significantly $(p<0.05)$ for nicotine and the lidocaine cream, and was smaller, but not significantly ( $p$ $=0.075)$ for the lidocaine patch. However, the further decrease in the drug level in the SC after the second clearance interval was not significant $(p>0.05$, repeated 

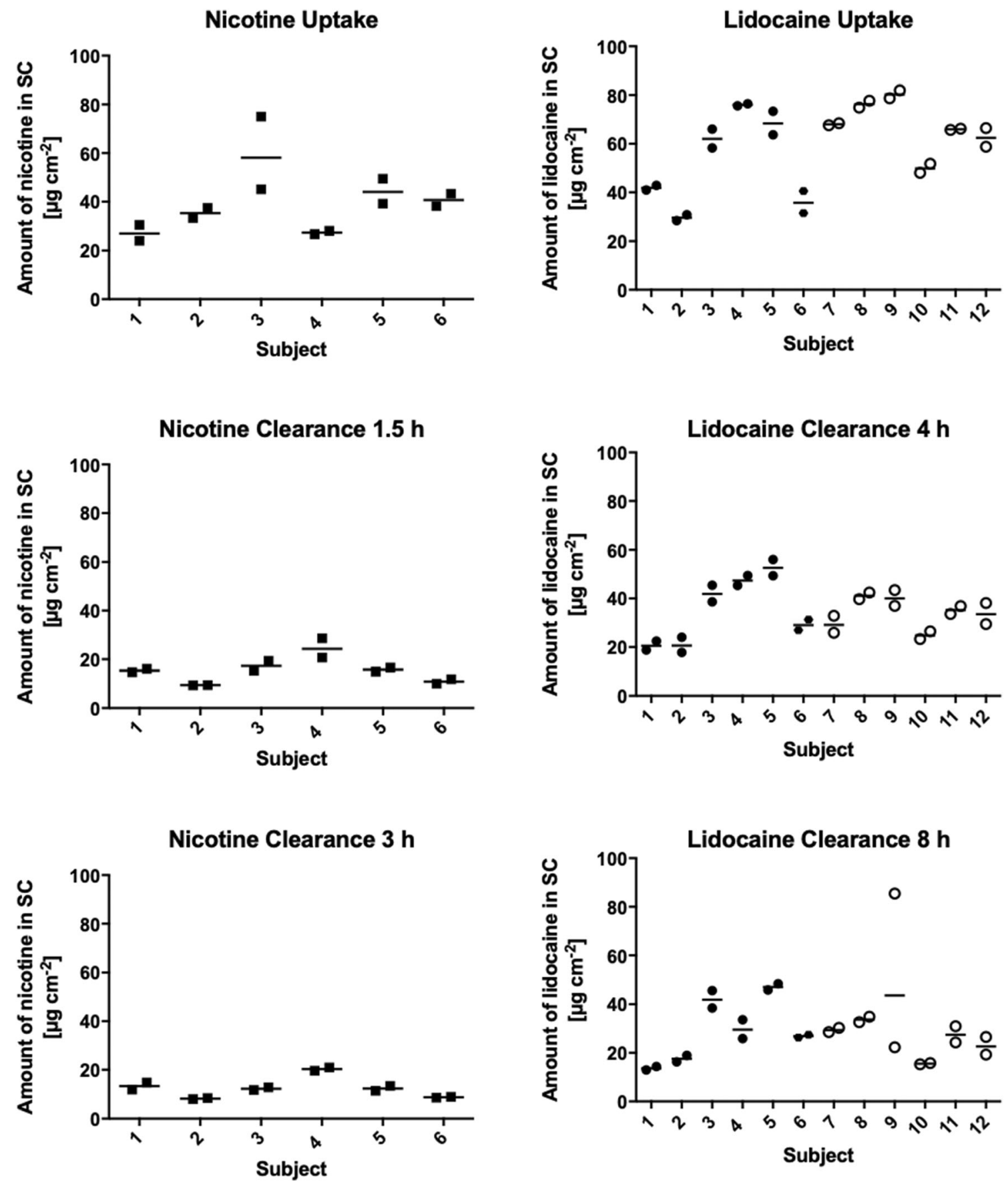

Fig. 2. Amounts of nicotine and lidocaine in the SC after uptake and clearance; duplicate measurements and the geometric mean of these values are shown (data from the patches, subjects 1-6, are indicated by solid symbols, those from lidocaine cream, subjects $7-12$, by open symbols)

measures one-way ANOVA with post hoc Bonferroni test); Fig. S2. When comparing the two lidocaine products, there was no significant difference in drug amounts in the SC either following uptake or after both of the two clearance periods (repeated measures one-way ANOVA with post hoc Bonferroni test).

The measured drug amounts in the SC after uptake and clearance periods (Table I) were then analysed by fitting the results for each subject to Eq. 3 (Fig. 3) and by deriving slope and intercept values corresponding to the first-order elimination rate constant $(\beta)$ of drug from the SC and the theoretical quantity of drug in the SC at the moment the uptake stopped $\left(\mathrm{M}_{\mathrm{UP}}\right)$. The average values of these metrics are presented in Table II. The linear regressions of the data were generally good with $r^{2}$ values across the six subjects studied for each drug/delivery system falling between 0.89 and 1.00 except for one subject in each of the nicotine and lidocaine patch studies $\left(r^{2}=0.82\right.$ and 0.75 , respectively) and two subjects in the lidocaine cream study $\left(r^{2}=0.74\right.$ and 0.65$)$. The 'predicted' $\mathrm{M}_{\mathrm{UP}}$ values were of course very similar to the experimental measurements reported in Table I. 
Table I. Amounts of Nicotine and Lidocaine in the SC after Uptake and Clearance; Geometric Mean (Lower-Upper 90\% Confidence Interval) of the Geometric Means of Duplicate Measurements from 6 Subjects per Treatment

\begin{tabular}{|c|c|c|c|c|c|}
\hline \multicolumn{2}{|l|}{ Nicotine patch } & \multicolumn{2}{|l|}{ Lidocaine patch } & \multicolumn{2}{|c|}{ Lidocaine cream } \\
\hline 2-h uptake & $37.0^{\mathrm{a}}(29.1-47.0)$ & 12-h uptake & $49.2(35.9-67.6)$ & 1-h uptake & $66.3(57.8-76.2)$ \\
\hline 1.5 -h clearance & $14.8(11.2-19.5)$ & 4-h clearance & $33.0(23.4-46.4)$ & 4-h clearance & $33.4(28.5-39.1)$ \\
\hline 3-h clearance & $12.0(9.2-15.6)$ & 8-h clearance & $26.9(18.1-39.9)$ & 8-h clearance & $27.2^{\mathrm{b}}(20.3-36.5)$ \\
\hline
\end{tabular}

${ }^{a} 35.5(29.4-42.8)$ if outlier for subject 3 excluded

${ }^{b} 24.4(19.4-30.5)$ if outlier for subject 9 excluded

The derived first-order elimination rate constants $(\beta)$ of drug from the SC, although relatively consistent, revealed a degree of inter-subject variability not uncommon for human, in vivo data associated with skin permeation. A one-way analysis of variance (ANOVA) of the $\beta$ results for the three delivery systems studied, followed by Tukey's multiple comparison test, revealed that the rate constant measured for nicotine was significantly greater than those determined for lidocaine, whether applied as a patch $(p<0.001)$ or as a cream $(p<0.01)$. In contrast, there was no significant difference in the $\beta$ values for lidocaine when administered as a patch or as a cream. A more detailed discussion and analysis of these results are provided in the Supplementary Information.

The fitted values of $\mathrm{M}_{\mathrm{UP}}$ and $\beta$ were then used, in accord with Eq. 4, to estimate the input rate $\left(R_{1}\right)$ of the drugs from the SC into the viable epidermis. These results, along with those for the rate constant and input rate derived for the shorter clearance interval ( $\beta_{\mathrm{CL} 1}$ and $R_{1, \mathrm{CL} 1}$, respectively) are presented in Table II. Values of $R_{2}$ were also assessed (see Table II) directly from the experimental measurements of drug in the SC following uptake and after the shorter clearance period, as indicated in Eq. 5 (6-9). It is worth emphasising that, when using the tape-stripping method, drug clearance from the SC is measured after the drug formulation has been removed from the skin surface. To estimate as closely as possible the input rate while the formulation is on the skin surface, the clearance rate should be measured as soon as possible after the drug product has been removed with the constraint that the clearance period must be long enough so that the drug amount in the SC decreases by a statistically significant amount compared to the amount after uptake.
It is immediately apparent that there is a close overlap between the values of $R_{1}$ and $R_{2}$ for each of the three drug formulations, suggesting that the two approaches are viable methods to provide a metric related to the rate at which a topically applied drug is able to reach the 'compartment' in which many targets for the treatment of dermatological disease are found. Statistically speaking (again based on a one-way ANOVA followed by Tukey's multiple comparison test), for both $R_{1}$ and $R_{2}$, the input rate of nicotine from the transdermal patch was significantly greater than that of lidocaine from its patch product; on the other hand, there was no difference between the values of either $R_{1}$ or $R_{2}$ when comparing the nicotine patch to lidocaine cream, or when comparing the lidocaine patch and cream formulations.

It is entirely plausible that a more mechanistic diffusion modelling approach may permit alternative strategies for the interpretation of the measurements presented here (as has been shown previously [7]). However, given the complexities of drug delivery from a topical dermatological formulation (including metamorphosis of the formulation and the impact of this transformation on drug delivery), in addition to the variability inherent to the quantitation of percutaneous absorption, a simpler measurement/analysis strategy that is informed by a knowledge of the diffusion process is better justified. This study must be viewed as an initial proof-of-concept, therefore, not the final word, and further work is required to explore these issues in the appropriate depth.

The in vitro skin permeation results are summarised in Fig. 4, where each panel provides both the cumulative penetration and the flux of the drug into the receptor compartment of the diffusion cell. After $5 \mathrm{~h}$, the average nicotine flux across the skin from the Nicotinell ${ }^{\circledR}$ patch over the final sampling interval ( 3.5 to $5 \mathrm{~h}$ ) was
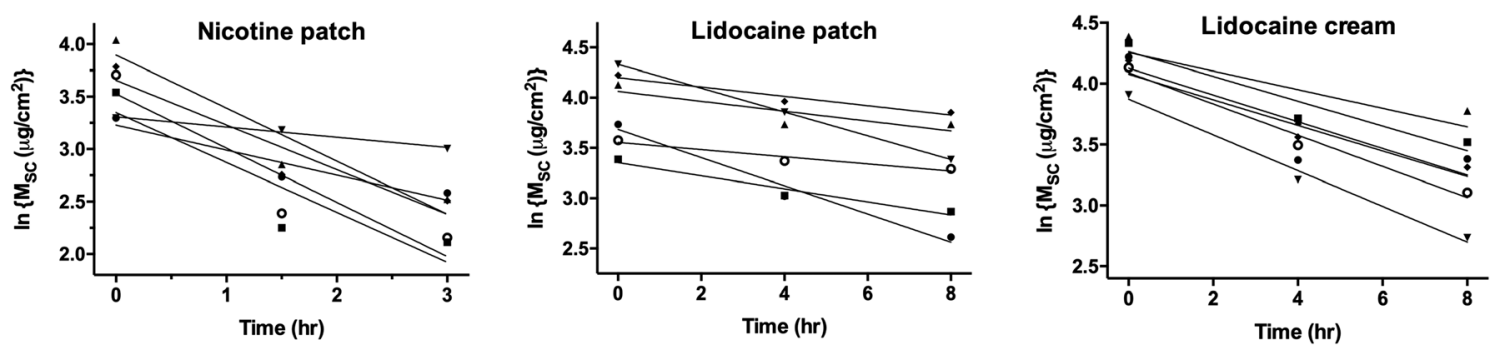

Fig. 3. Drug clearance from the stratum corneum (SC) plotted according to Eq. 3 after removal of the applied formulation. Values of $\beta$ and $\mathrm{M}_{\mathrm{UP}}$ were deduced from the slope and y-axis intercept at $t=0$ of the linear regressions indicated (each line and symbol reflecting the data from the 6 subjects studied for each formulation) 
Table II. Dermal Pharmacokinetic Metrics Deduced from the Amounts of Drug Measured in the SC after Uptake and Clearance (Values Shown Are mean $\pm S \mathrm{D} ; n=6$ )

\begin{tabular}{|c|c|c|c|}
\hline Dermal pharmacokinetic metric & Nicotine patch ${ }^{\mathrm{a}}$ & Lidocaine patch & 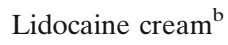 \\
\hline $\begin{array}{l}\mathrm{M}_{\mathrm{UP}} \text { 'predicted' }\left(\mu \mathrm{g} \mathrm{cm}^{-2}\right)^{\mathrm{c}} \\
\beta\left(\mathrm{h}^{-1}\right)^{\mathrm{d}} \\
R_{1}=\beta \times \mathrm{M}_{\mathrm{UP}}\left(\mu \mathrm{g} \mathrm{cm}^{-2} \mathrm{~h}^{-}\right)^{\mathrm{e}} \\
\beta_{\mathrm{CL} 1}\left(\mathrm{~h}^{-1}\right)^{\mathrm{f}} \\
R_{1, \mathrm{CL} 1}=\beta \times \mathrm{M}_{\mathrm{UP}}\left(\mu \mathrm{g} \mathrm{cm}^{-2} \mathrm{~h}^{-1}\right)^{\mathrm{g}} \\
R_{2}=\left(\mathrm{M}_{\mathrm{UP}}-\mathrm{M}_{\mathrm{CL}}\right) / \Delta \mathrm{t}\left(\mu \mathrm{g} \mathrm{cm}^{-2} \mathrm{~h}^{-1}\right)^{\mathrm{h}}\end{array}$ & $\begin{array}{l}33.8 \pm 9.0 \\
0.37 \pm 0.17 \\
13.5 \pm 8.1 \\
0.61 \pm 0.32 \\
25.5 \pm 16.2 \\
15.2 \pm 8.9\end{array}$ & $\begin{array}{l}50.7 \pm 19.0 \\
0.076 \pm 0.043 \\
3.9 \pm 2.9 \\
0.100 \pm 0.045 \\
5.3 \pm 2.8 \\
4.2 \pm 2.1\end{array}$ & $\begin{array}{l}61.7 \pm 8.5 \\
0.111 \pm 0.024 \\
6.7 \pm 0.8 \\
0.172 \pm 0.021 \\
11.5 \pm 2.3 \\
8.3 \pm 1.5\end{array}$ \\
\hline \multicolumn{4}{|c|}{$\begin{array}{l}{ }^{a} \text { If the outlier is excluded, } \mathrm{M}_{\mathrm{UP}} \text { 'predicted' }=32.3 \pm 6.2, \beta=0.36 \pm 0.16, R_{1}=12.2 \pm 6.3 ; \beta_{\mathrm{CL} 1}=0.58 \pm 0.31, R_{1, \mathrm{CL} 1}=22.5 \pm 13.3 \text {, and } R_{2}=13.8 \pm \\
7.3 \\
{ }^{b} \text { If the outlier is excluded, } \mathrm{M}_{\mathrm{UP}} \text { 'predicted' }=63.1 \pm 10.7, \beta=0.125 \pm 0.024, R_{1}=7.9 \pm 2.4 ; \beta_{\mathrm{CL} 1}, R_{1, \mathrm{CL} 1} \text { and } R_{2} \text { are unchanged } \\
{ }^{c} \text { Drug amount predicted in the } \mathrm{SC} \text { at the end of the uptake period from the intercept of the linear regressions in Fig. } 3 \text { according to Eq. } 3 \\
{ }^{d} \text { First-order clearance rate constant of drug from the SC calculated from the slope of the linear regressions in Fig. } 3 \text { according to Eq. } 3 \\
{ }^{e} \text { Input rate of the drug from the SC into the viable skin at the end of the uptake period according to Eq. } 4 \text { if } \beta \text { is constant for the entire } \\
\text { clearance interval } \\
{ }^{f} \text { First-order clearance rate constant of drug from the SC for the shorter clearance time calculated according to Eq. } 5 \\
{ }^{g} \text { Input rate of the drug from the SC into the viable skin at the end of the uptake period estimated from the shorter clearance time and the } \\
\text { geometric mean value for } \mathrm{M}_{\mathrm{UP}} \text { according to Eq. } 5 \\
{ }^{2} \text { Input rate of the drug from the SC into the viable skin estimated from the difference between drug amounts in SC after uptake and after a } \\
\text { period }(\Delta \mathrm{t}) \text { of clearance according to Eq. } 6\end{array}$} \\
\hline
\end{tabular}

$23.7( \pm 7.5) \mu \mathrm{g} \mathrm{cm}^{-2} \mathrm{~h}^{-1}$. Given that the $10 \mathrm{~cm}^{2}$ system is labelled to deliver $7 \mathrm{mg}$ over $24 \mathrm{~h}$, the expected in vivo performance of the patch corresponds to a flux of $29 \mu \mathrm{g} \mathrm{cm}^{-2} \mathrm{~h}^{-1}$, and suggests a reasonable in vitro-in vivo correlation, therefore. The in vitro flux results are also close to the in vivo input rate for the first clearance interval $R_{1, \mathrm{CL} 1}(25.5 \pm 16.2) \mu \mathrm{g} \mathrm{cm}{ }^{-2} \mathrm{~h}^{-1}$ and within a factor of 2 of the in vivo values of $R_{1}$ and $R_{2}$ for nicotine discussed above (13.5 \pm 8.1 and $15.2 \pm 8.9 \mu \mathrm{g} \mathrm{cm} \mathrm{cm}^{-2} \mathrm{~h}^{-1}$, respectively) which were determined following only $2 \mathrm{~h}$ of patch wear. The average in vitro flux deduced over the final hour of a 2-h application was 14.2 ( \pm 6.0) $\mu \mathrm{g} \mathrm{cm} \mathrm{ch}^{-1}$. The Summary of Product Characteristics (SmPC) for this matrix system (27) states that, 'following a single application... there is an initial 1-2 hour delay followed by a progressive rise in nicotine plasma concentrations, with a plateau attained at about 8-10 hours after application'. While it is true that the rate of drug release from a matrix system will initially be fast and then slow down over time, the presence of the rate-limiting SC causes the delivery into the patient (or into an IVPT receptor phase, or into the viable skin) to follow the profile described in the product's SmPC. For that reason, which is well-understood in the field, the comparison of the nicotine input rate, derived from SC sampling, with the labelled, average flux of $7 \mathrm{mg}$ per $24 \mathrm{~h}$ is reasonable.

The Summary of Product Characteristics (SmPC) of the Versatis ${ }^{\circledR}$ medicated plaster used (28) indicates that the 140 $\mathrm{cm}^{2}$ patch contains a total of $700 \mathrm{mg}$ of drug, corresponding to a loading of $5 \mathrm{mg} \mathrm{cm}^{-2}$. The SmPC also highlights that the plaster, when worn for the recommended $12 \mathrm{~h}$, delivers systemically 'about $3 \pm 2 \%$ of the total applied lidocaine dose', i.e. the equivalent of $150( \pm 100) \mu \mathrm{g} \mathrm{cm}^{-2}$, at an average flux of $12.5( \pm 8.3) \mu \mathrm{g} \mathrm{cm}^{-2} \mathrm{~h}^{-1}$. The in vitro results in Fig. 4 agree quite well with this information: cumulative permeation in $12 \mathrm{~h}$ was nearly $70 \mu \mathrm{g} \mathrm{cm}^{-2}$ and the terminal flux when the patch was removed was $\sim 8 \mu \mathrm{g} \mathrm{cm}^{-2} \mathrm{~h}^{-1}$. The in vivo input rate (whether $R_{1}$ or $R_{2}$ ) deduced from the SC sampling experiment (Table II) was about half (and modestly larger for $\left.R_{1, \mathrm{CL} 1}\right)$ that measured in vitro but still within the range of values reported in the SmPC. It is important to point out that the sample sizes for both the in vivo and in vitro experiments are small, reducing the power of the comparisons with the reported patch results; better agreement might be observed with larger sample sizes.

Lidocaine uptake into the SC in vivo from the commercial cream following a 1-h application was about $70 \mu \mathrm{g} \mathrm{cm}^{-2}$ (Table I) and the deduced input rate to the viable epidermis was similar for $R_{1}\left(6.7 \pm 0.8 \mu \mathrm{g} \mathrm{cm}^{-2} \mathrm{~h}^{-1}\right)$ and $R_{2}(8.3 \pm 1.5 \mu \mathrm{g}$ $\left.\mathrm{cm}^{-2} \mathrm{~h}^{-1}\right)$ and a little larger for the first clearance period (11.5 $\left.\pm 2.3 \mu \mathrm{g} \mathrm{cm}^{-2} \mathrm{~h}^{-1}\right)$. The in vitro skin penetration experiments reinforced the apparent rapid uptake of the drug into the skin compared to the lidocaine patch, with lag times, respectively,
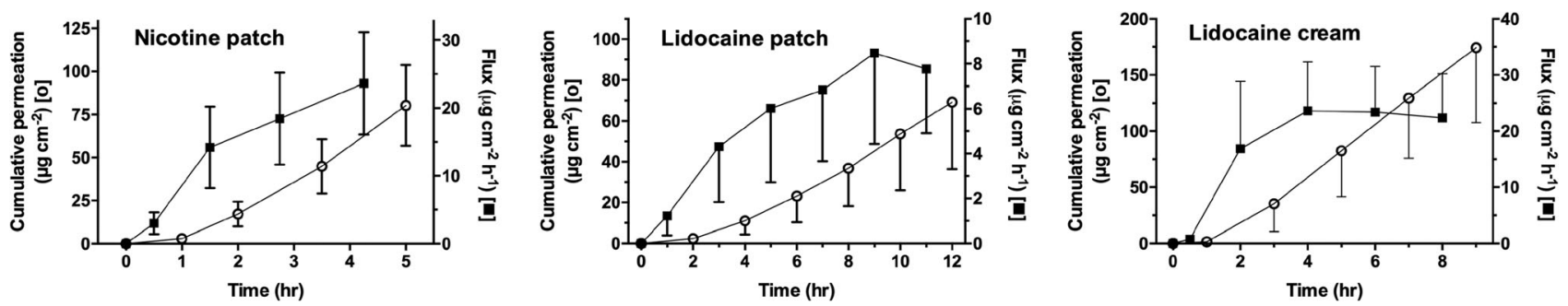

Fig. 4. In vitro skin permeation results for the formulations considered. Data (mean $\pm S \mathrm{D} ; n=6$ for nicotine and $n=7$ for lidocaine) are presented as the cumulative amount of drug absorbed (open symbols, left axis) and as the flux, plotted at the mid-point of the sampling interval, as a function of time (closed symbols, right axis) 
Table III. Estimation of Drug Concentrations at the Site of Action in the Viable Skin (C*) from SC Sampling Results for Nicotine Delivered from a Patch and for Lidocaine Delivered from a Medicated Plaster and from a Cream

\begin{tabular}{lllll}
\hline Drug (delivery system) & $\left(\mathrm{M}_{\mathrm{UP}}-\mathrm{M}_{\mathrm{CL}}\right) / \Delta \mathrm{t}\left(\mu \mathrm{g} \mathrm{cm}{ }^{-2} \mathrm{~h}^{-1}\right)^{\mathrm{a}}$ & $\mathrm{D}_{\mathrm{D}}\left(\mathrm{cm}^{2} \mathrm{~h}^{-1}\right)^{\mathrm{b}}$ & $\mathrm{P}_{\mathrm{D}}\left(\mathrm{cm} \mathrm{h}^{-1}\right)^{\mathrm{c}}$ & 1.015 \\
\hline Nicotine (patch) & 15.2 & 0.0101 & 0.757 & 15.0 \\
Lidocaine (plaster) & 4.2 & 0.0076 & 0.757 & 5.6 \\
Lidocaine (cream) & 8.3 & 0.0076 & $\left.0.75 \mathrm{~cm}^{-3}\right)$ \\
\hline
\end{tabular}

${ }^{a}$ Average input flux from Table II (defined as $R_{2}$ ) determined from SC sampling experiments

${ }^{b}$ Average value from two algorithms proposed by Krestos et al. (10)

${ }^{c} \mathrm{P}_{\mathrm{D}}=\mathrm{D}_{\mathrm{D}} / \mathrm{h}_{\mathrm{D}}$, where $\mathrm{h}_{\mathrm{D}}$ is assumed to be $100 \mu \mathrm{m}$ (6)

of approximately 1.5 and $4 \mathrm{~h}$, a fact quite possibly attributable to the significant presence of two excipients in the formulation, specifically propylene glycol (at 7.5\% w/w) and benzyl alcohol $(1.5 \% \mathrm{w} / \mathrm{w})$. This was also evident from the spatial distribution of lidocaine in the SC as shown in Fig. S1 (Supplementary Information); while the drug was fairly evenly distributed after patch wear, the short duration of cream application resulted in an initially high loading and a steep concentration gradient. It seems reasonable to speculate, upon application of the cream to the skin, that the transformation, or 'metamorphosis', of the formulation may lead to a rapid input of drug into the SC as these small molecular weight excipients themselves are taken up and/or lost by evaporation, thereby both (potentially) acting as penetration enhancers and increasing the drug's thermodynamic activity in the evolving residual phase remaining on the skin surface. Notably, lidocaine flux from the cream reached a maximum at about $3 \mathrm{~h}$ that was maintained until the end of the 9-h experiment. Given the relatively large dose of cream applied, it is reasonable to anticipate that a steady flux of the drug would have been sustained for a further period of time.

As a more general point with respect to the lidocaine cream data, it is strongly recommended that estimates of drug concentration in the target tissue from a topical dermatological formulation should be measured from doses that are relevant to their intended use. While acknowledging that the lidocaine cream dose is high relative to that of other topical products, it is worth noting that problems with dose precision and/or analytical sensitivity have not been experienced when using formulations that are applied in much smaller quantities. For example, a study that measured econazole in tape-stripped SC involved dosing with $4.5 \mathrm{mg} / \mathrm{cm}^{2}$ of $1 \%$ econazole nitrate creams (7); similarly, a diclofenac investigation (8) used the recommended doses of the formulations studied (i.e. either 10 or $20 \mathrm{mg} / \mathrm{cm}^{2}$ ).

It should also be emphasised that the determination of drug input rate to the viable tissue using skin sampling by tape-stripping does not require measurement of SC mass collected. The protocol is designed, by use of the TEWL measurements, to collect most of the SC (and thereby most of the drug in the SC), which reduces the site-to-site variability (and also minimises investigator-to-investigator variability in studies with multiple investigators). In this study, the SC masses collected on the tape-strips from the three products and three measurement times were consistent (Table S1) and showed no statistically significant differences $(p<0.05)$. Overall, about $75 \%$ of the SC thickness was removed by the tape-strips, corresponding to approximately $90 \%$ of the drug (see Supplementary Information).
Finally, as recently reported for acyclovir (6), the experimentally determined 'input' fluxes from the SC sampling experiments reported here can be equated with the product of the free drug concentration $\left(\mathrm{C}^{*}\right)$ at the site of action (the sub-SC 'compartment', exemplified by the basal epidermis) and a heterogeneous rate constant $\left(\mathrm{P}_{\mathrm{D}}\right)$, describing drug clearance from the site $(12-16) ; \mathrm{P}_{\mathrm{D}}=\mathrm{D}_{\mathrm{D}} / \mathrm{h}_{\mathrm{D}}$, where $\mathrm{D}_{\mathrm{D}}$ is the drug's diffusivity in the dermis, and is calculable from molecular weight with available algorithms (10), and $h_{D}$ is the distance that a drug must diffuse from the basal epidermis to the microcirculation where it is cleared from the skin. In this way, it is possible to estimate values of $\mathrm{C}^{*}$ achieved for nicotine and lidocaine, as shown in Table III.

It is perhaps unsurprising that the estimations of $C^{*}$ for nicotine and lidocaine, which may be considered as relatively good skin penetrants, are considerably larger than that assessed for the poorly absorbed acyclovir $\left(\sim 0.04 \mu \mathrm{g} \mathrm{cm}^{-3}\right)(6)$.

\section{CONCLUSIONS}

The research described in this paper aimed to further address the challenge of assessing the topical bioavailability of a drug at its site of action in the skin. Specifically, experiments were designed to test the hypothesis that interrogation of drug levels in the stratum corneum (SC) in vivo can permit the 'input rate' into the underlying, living skin 'compartment' (where many dermatological disease targets are found) to be deduced. The results demonstrate that the interpretation of drug amounts in the SC after periods of uptake, when the formulation is in contact with the skin, and after periods of 'clearance', post-removal of the formulation, yield deduced 'input rates' that are consistent with known product performance and in broad agreement with conventional in vitro skin permeation test measurements. This further validation of the SC sampling methodology provides additional evidence to support its application to the assessment of local, topical bioavailability of drugs that act within the skin. The in vivo nature of the measurements suggests, furthermore, that the approach may find useful application in the determination of topical drug product bioequivalence for which an approach (or a 'tool-kit' of approaches, which might include more specialised microdialysis and spectroscopic techniques, for example) to avoid the need for clinical end-point studies is an important current goal.

\section{SUPPLEMENTARY INFORMATION}

The online version contains supplementary material available at https://doi.org/10.1208/s12248-021-00571-3. 


\section{ACKNOWLEDGEMENTS}

M. Hoppel and M.A.M. Tabosa acknowledge the financial support of the Austrian Science Fund (FWF) J3754-B30 (Erwin-Schrödinger Fellowship J3754-B30) and the CAPES Foundation (Brazilian 'Ciência sem Fronteiras' programme), respectively.

\section{FUNDING}

This study was funded by The Leo Foundation (grant number LF16117).

Open Access This article is licensed under a Creative Commons Attribution 4.0 International License, which permits use, sharing, adaptation, distribution and reproduction in any medium or format, as long as you give appropriate credit to the original author(s) and the source, provide a link to the Creative Commons licence, and indicate if changes were made. The images or other third party material in this article are included in the article's Creative Commons licence, unless indicated otherwise in a credit line to the material. If material is not included in the article's Creative Commons licence and your intended use is not permitted by statutory regulation or exceeds the permitted use, you will need to obtain permission directly from the copyright holder. To view a copy of this licence, visit http://creativecommons.org/licenses/by/4.0/.

\section{REFERENCES}

1. Mitragotri S, Anissimov YG, Bunge AL, Frasch HF, Guy RH, Hadgraft J, et al. Mathematical models of skin permeability: an overview. Int J Pharm. 2011;418:115-29. https://doi.org/10.1016/ j.ijpharm.2011.02.023.

2. Bodenlenz M, Tiffner K, Raml R, Augustin T, Dragatin C, Birngruber T, et al. Open flow microperfusion as a dermal pharmacokinetic approach to evaluate topical bioequivalence. Clin. Pharmacokin. 2017;56:91-8. https://doi.org/10.1007/s40262016-0442-z.

3. Kezic S. Methods for measuring in-vivo percutaneous absorption in humans. Hum Exp Toxicol. 2008;27:289-95. https:// doi.org/10.1177/0960327107085825.

4. Franzen L, Windbergs M. Applications of Raman spectroscopy in skin research-from skin physiology and diagnosis up to risk assessment and dermal drug delivery. Adv Drug Deliv Rev. 2015;89:91-104. https://doi.org/10.1016/j.addr.2015.04.002.

5. Raney SG, Franz TJ, Lehman PA, Lionberger R, Chen ML. Pharmacokinetics-based approaches for bioequivalence evaluation of topical dermatological drug products. Clin Pharmacokinet. 2015;54:1095-106. https://doi.org/10.1007/ s40262-015-0292-0.

6. Pensado A, Chiu WS, Cordery SF, Rantou E, Bunge AL, Delgado-Charro MB, et al. Stratum corneum sampling to assess bioequivalence between topical acyclovir products. Pharm Res. 2019;36:180. https://doi.org/10.1007/s11095-019-2707-3.

7. N'Dri-Stempfer B, Navidi WC, Guy RH, Bunge AL. Improved bioequivalence assessment of topical dermatological drug products using dermatopharmacokinetics. Pharm Res. 2009;26:316-28. https://doi.org/10.1007/s11095-008-9742-9.

8. Cordery SF, Pensado A, Chiu WS, Shehab MZ, Bunge AL, Delgado-Charro MB, et al. Topical bioavailability of diclofenac from locally-acting, dermatological formulations. Int J Pharm. 2017;529:55-64. https://doi.org/10.1016/j.ijpharm.2017.06.063.

9. de Araujo TP, Fittipaldi IM, Bedor DCG, Duarte ML, Cordery SF, Guy RH, et al. Topical bio (in) equivalence of metronidazole formulations in vivo. Int J Pharm. 2018;541:167-72. https:// doi.org/10.1016/j.ijpharm.2018.02.032.

10. Kretsos K, Miller MA, Zamora-Estrada G, Kasting GB. Partitioning, diffusivity and clearance of skin permeants in mammalian dermis. Int J Pharm. 2008;346:64-79. https://doi.org/ 10.1016/j.ijpharm.2007.06.020.

11. Maciel Tabosa MA, Hoppel M, Bunge AL, Guy RH, DelgadoCharro MB. Predicting topical drug clearance from the skin. Drug Deliv Transl Res. 2020. https://doi.org/10.1007/s13346-02000864-8.

12. Mehta SC, Afouna MI, Ghanem AH, Higuchi WI, Kern ER. Relationship of skin target site free drug concentration $\left(\mathrm{C}^{*}\right)$ to the in vivo efficacy: an extensive evaluation of the predictive value of the $\mathrm{C}^{*}$ concept using acyclovir as a model drug. J Pharm Sci. 1997;86:797-801. https://doi.org/10.1021/js960515h.

13. Imanidis G, Song WQ, Lee PH, Su MH, Kern ER, Higuchi WI. Estimation of skin target site acyclovir concentrations following controlled (trans)dermal drug delivery in topical and systemic treatment of cutaneous HSV-1 infections in hairless mice. Pharm Res. 1994;11:1035-41. https://doi.org/10.1023/ a:1018995606568.

14. Afouna MI, Mehta SC, Ghanem AH, Higuchi WI, Kern ER, De Clercq E, et al. Assessment of correlation between skin target site free drug concentration and the in vivo topical antiviral efficacy in hairless mice for (E)-5-(2-bromovinyl)-2'deoxyuridine and acyclovir formulations. J Pharm Sci. 1998;87:917-21. https://doi.org/10.1021/js980052a.

15. Patel PJ, Ghanem AH, Higuchi WI, Srinivasan V, Kern ER. Correlation of in vivo topical efficacies with in vitro predictions using acyclovir formulations in the treatment of cutaneous HSV1 infections in hairless mice: an evaluation of the predictive value of the $C^{*}$ concept. Antivir Res. 1996;29:279-86. https:// doi.org/10.1016/0166-3542(96)80225-7.

16. Lee PH, Su M, Ghanem A, Inamori T, Kern ER, Higuchi WI. An application of the $\mathrm{C}^{*}$ concept in predicting the topical efficacy of finite dose acyclovir in the treatment of cutaneous HSV-1 infections in hairless mice. Int J Pharm. 1993;93:139-52. https://doi.org/10.1016/0378-5173(93)90172-C.

17. Goyal R, Macri LK, Kaplan HM, Kohn J. Nanoparticles and nanofibers for topical drug delivery. J Control Release. 2016;240:77-92. https://doi.org/10.1016/j.jconrel.2015.10.049.

18. Gammaitoni AR, Alvarez NA, Galer BS. Safety and tolerability of the lidocaine patch $5 \%$, a targeted peripheral analgesic: a review of the literature. J Clin Pharmacol. 2003;43:111-7. https:// doi.org/10.1177/0091270002239817.

19. Surber C, Smith WE. The mystical effects of dermatological vehicles. Dermatology. 2005;210:157-68. https://doi.org/10.1159/ 000082572.

20. Sekkat N, Guy RH. Biological models to study skin permeation. In: Testa B, Van de Waterbeemd H, Folkers G, Guy RH, editors. Pharmacokinetic optimisation in drug research. Zürich: 155Y172, Wiley-VCH and VHCA; 2001. p. 155-72. https:// doi.org/10.1002/9783906390437.ch10.

21. Anderson RL, Cassidy JM. Variations in physical dimensions and chemical composition of human stratum corneum. J Invest Dermatol. 1973;1:30-2. https://doi.org/10.1111/15231747.ep12674117.

22. Electronic Medicines Compendium. LMX4 Lidocaine 4\% w/w Cream. Summary of product characteristics. https:// www.medicines.org.uk/emc/product/5938/smpc\#gref. Accessed January 10, 2021.

23. Bunge AL, Cleek RL, Vecchia BE. A new method for estimating dermal absorption from chemical exposure. 3 . Compared with steady-state methods for prediction and data analysis. Pharm Res. 1995;12:972-82. https://doi.org/10.1023/ a:1016298012408. 
24. Ho H, Chien YW. Kinetic evaluation of transdermal nicotine delivery systems. Drug Dev Ind Pharm. 1993;19:295-313. https:// doi.org/10.3109/03639049309038769.

25. Kuswahyuning R, Roberts MS. Concentration dependency in nicotine skin penetration flux from aqueous solutions reflects vehicle induced changes in nicotine stratum corneum retention. Pharm Res. 2014;31:1501-11. https://doi.org/10.1007/s11095-013-1256-4.

26. Analytical Methods Committee. Using the Grubbs and Cochran tests to identify outliers. Anal Methods. 2015;7:7948-50.

27. Electronic Medicines Compendium. Nicotinell TTS 10 transdermal patches. Summary of product characteristics. https:// www.medicines.org.uk/emc/product/390/smpc\#gref. Accessed 6 Feb 2021.

28. Electronic Medicines Compendium. Versatis 5\% medicated plaster. Summary of product characteristics. https:// www.medicines.org.uk/emc/medicine/19291. Accessed 6 Feb 2021.

Publisher's Note Springer Nature remains neutral with regard to jurisdictional claims in published maps and institutional affiliations. 